\title{
STRUCTURE AND MAGNETISM OF MBE-GROWN Co/Cu MULTILAYERS
}

\author{
L.T. Baczewski, A. Wawro, J. Domagala, J. Pelka, A. Szewczyk \\ AND A. NABIAEEK
}

Institute of Physics, Polish Academy of Sciences

Al. Lotników 32/46, 02-668 Warsaw, Poland

\begin{abstract}
Structural and magnetic properties of $\mathrm{Co} / \mathrm{Cu}$ multilayers deposited in the ultra-high vacuum molecular beam epitaxy system on glass substrates with different modulations periods were investigated. A structural characterization was performed by means of RHEED and Auger spectroscopy (in situ), small angle $\mathrm{X}$-ray reflectivity and scanning tunneling microscopy. The samples obtained have a textured, polycrystalline layered structure for deposition at room temperature. Magnetization and in-plane magnetoresistance measurements were performed as a function of $\mathrm{Cu}$ and Co layer thicknesses. An influence of different buffers and of interface quality on magnetic properties was investigated.
\end{abstract}

PACS numbers: $75.30 .-\mathrm{m}, 75.30 . \mathrm{Gw}, 75.70 .-\mathrm{i}$

\section{Introduction}

The magnetotransport behaviour of $\mathrm{Co} / \mathrm{Cu}$ multilayers grown by molecular beam epitaxy (MBE) has recently generated some controversy. The problem concerns the appearance of giant magnetoresistance (GMR) in multilayers that have little [1,2] or no [3] evidence for antiferromagnetic (AF) coupling between Co layers which is up to now a well-established mechanism responsible for GMR in magnetic multilayers through spin dependent scattering. Some authors have put forward sample structural defects as a possible explanation for a lack of antiferromagnetic coupling, for example it was suggested that stacking faults [4] and pinholes [5] may lead to ferromagnetic bridging across neighbouring layers. However, ferromagnetic bridging, by definition, suppresses antiferromagnetic (AF) coupling, therefore it should suppress the spin dependent GMR effect as well. In this paper the GMR investigations for polycrystalline $\mathrm{Co} / \mathrm{Cu}$ multilayers deposited on amorphous substrates (glass) are presented. Such films have obviously more structural defects than epitaxial samples but still magnetic coupling oscillations and the GMR effect are clearly present. 


\section{Experimental details}

The samples were deposited on glass substrates in MBE EVA 32 Riber system in UHV of $10^{-10}$ Torr using electron guns. A configuration of the studied samples was defined as glass/buffer $/(\operatorname{CoX} \mathrm{Cu} Y) n$, where $20<n<50$ is a number of $\mathrm{Co} / \mathrm{Cu}$ bilayers, $5<X, Y<100$ - thicknesses in $\AA$ of Co and $\mathrm{Cu}$ individual layers. Cobalt or copper buffer layers of different thicknesses between 50 and $300 \AA$ were used. The deposition rates of the order of $0.3-0.5 \AA / \mathrm{s}$ were controlled by Leybold Hereaus electron impact emission spectrometer Sentinel III. In situ characterization was performed by RHEED and Auger spectroscopy, a textured, polycrystalline structure was found with no sign of oxygen contamination. Grazing incidence X-ray reflectometry was performed in order to check the thickness of the layers and interface quality by use of Philips MRD diffractometer with the conventional $\mathrm{Cu} K_{\alpha}$ radiation source. VSM magnetometer was used for magnetization measurements and the GMR effect was measured using the four-probe method.

\section{Results and discussion}

The grazing-incidence X-ray reflectometry is a valuable tool to provide the important information about quality and configuration of obtained multilayers. The experimental spectra were analyzed by a comparison with the theoretical model calculated from the recursive algorithm based on the Fresnel equations [6]. Multilayers spectra confirmed the modulated structure by the presence of at least two Bragg peaks (see Fig. 1). From the period of Kiessig fringes the thickness of $\mathrm{Co} / \mathrm{Cu}$ bilayer was obtained. The comparison between experimental and theoretical spectra (a detailed analysis will be published elsewhere) permitted determination of interface roughness of $7 \AA$ in our best samples. The surface roughness of copper layer deposited at room temperature was lower than that deposited at $200^{\circ} \mathrm{C}$. The latter result and also the presence of a modulated structure in studied $\mathrm{Co} / \mathrm{Cu}$ multilayers was corroborated by STM scanning tunneling microscopy (see our another paper of this conference [7]).

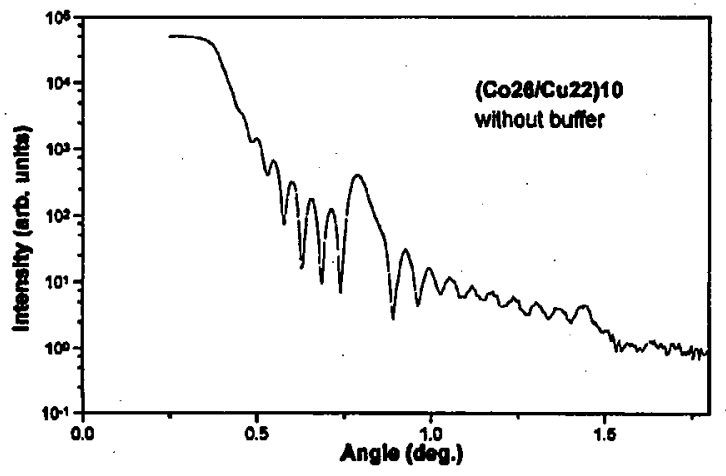

Fig. 1. Grazing incidence X-ray reflectometry spectrum for Co25/Cu20 multilayer. 

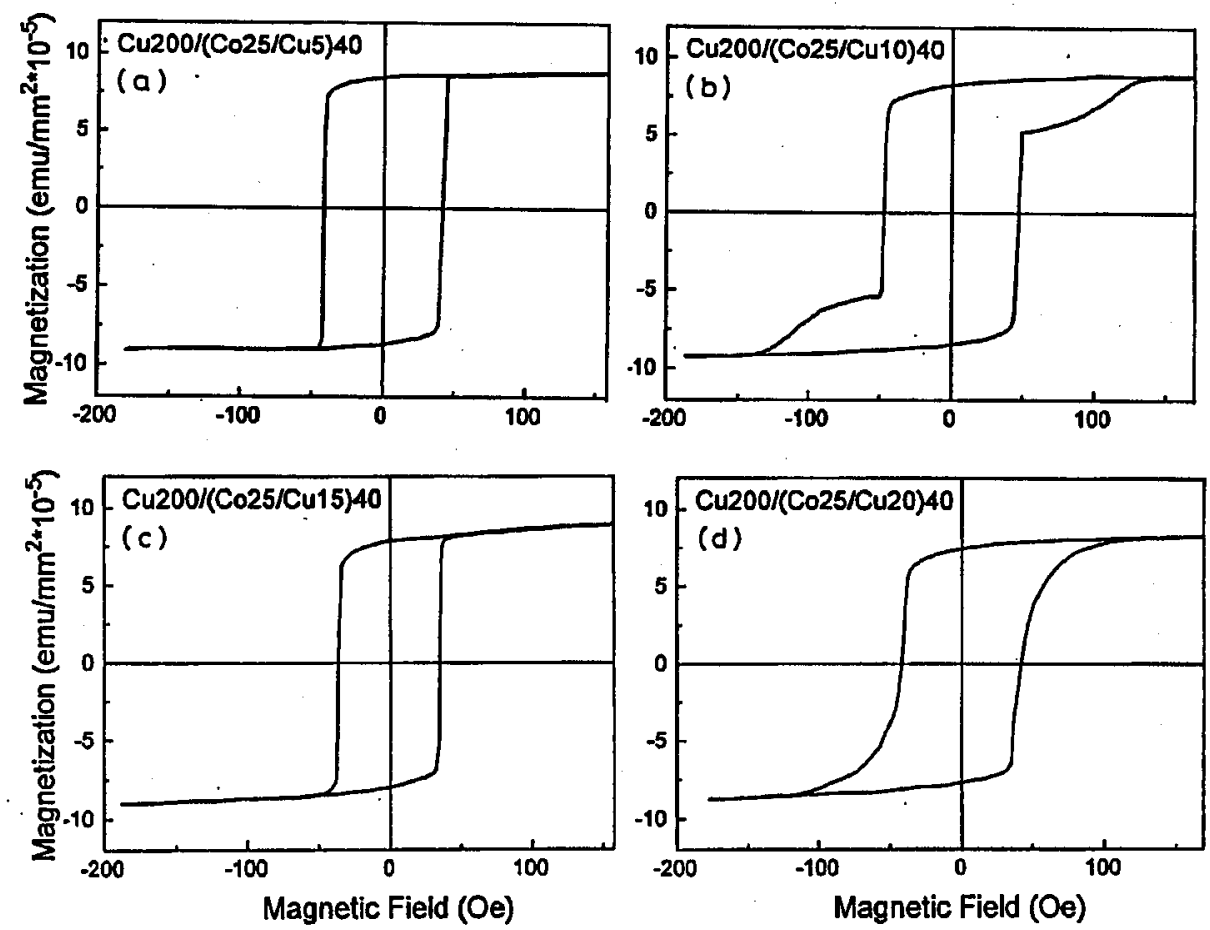

Fig. 2. Hysteresis loops for $\mathrm{Cu} 200 /(\mathrm{Co} 25 / \mathrm{Cu} X) 40$ multilayers, where $X=5 \AA$ (a), $10 \AA$ (b), $15 \AA$ (c), $20 \AA$ (d). The measurements were performed at room temperature with the applied field in the film plane.

Hysteresis loops of Co25/CuX multilayers deposited on Cu $200 \AA$ buffer where $X=5,10,15$ and $20 \AA$ are presented in Fig. 2. An oscillatory character of antiferromagnetic coupling between the adjacent Co layer across the copper interlayer can be seen clearly. AF coupling is absent for 5 and $15 \AA$ but present for 10 and $20 \AA$ thicknesses of $\mathrm{Cu}$ interlayer. The AF coupling is stronger for the first peak around $10 \AA$ than for the second peak around $20 \AA$ what is consistent with the previously reported data, e.g. [8]. Magnetoresistance measurements for these samples were however disappointing because we got only the $2 \%$ GMR effect for $\mathrm{Co} 25 / \mathrm{Cu} 10$ sample and $0.75 \%$ for $\mathrm{Co} 25 / \mathrm{Cu} 5$ one at room temperature. X-ray and STM measurements revealed that the surface roughness of $\mathrm{Cu}$ is considerably higher than that of Co layer so we decided to try cobalt as a buffer layer. Buffer roughness deteriorated the quality of modulated structure and it was reflected by low GMR values.

Multilayer of (Co25/Cu20)10 deposited directly on a glass substrate had no higher GMR value $(0.56 \%)$ at room temperature than the same sample deposited on $200 \AA \mathrm{Cu}$ buffer. However, the situation has changed when we used Co buffer layers. Different thicknesses of Co buffer layers were tried (50,100 and $200 \AA)$ for the Co25/Cu18 multilayer. Co buffer thickness had a clear influence on a shape of 
hysteresis loop - changing coercivity, remanence and coupling. The sample with $200 \AA$ Co buffer showed an almost rectangular hysteresis loop while for the samples with 50 and $100 \AA$ Co buffer AF coupling features were present. The sample with $50 \AA$ Co buffer layer had smaller remanence magnetization and coercivity than for the one with $100 \AA$ Co buffer. Also magnetoresistance measurements have given the highest value for the sample Co25/Cu18 with $100 \AA$ Co buffer layer $(5.2 \%$ at room temperature). For the same multilayer on 50 and $200 \AA$ Co buffer layer the values of $3.6 \%$ and $2.1 \%$ were obtained respectively for magnetoresistive effect. It seems that the too thin buffer layer did not smooth enough the substrate roughness and the too thick buffer introduced the important shunting effect to the measured value.

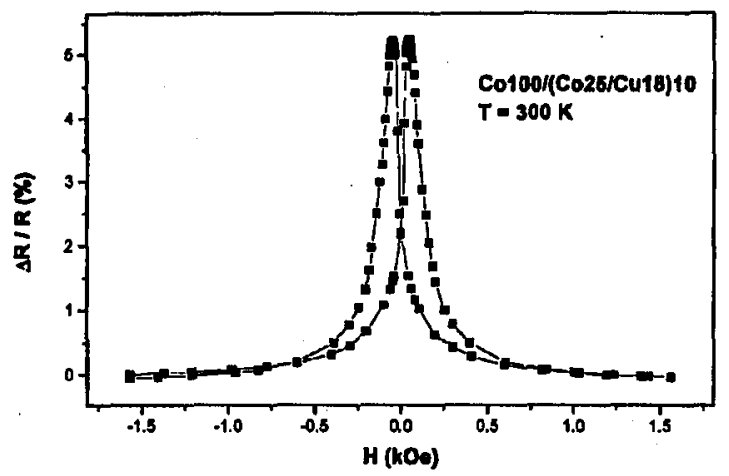

Fig. 3. A magnetoresistance loop for Co100/(Co25/Cu18)10 multilayer deposited on glass substrate. A magnetic field was applied perpendicular to the current direction.

A typical magnetoresistance loop versus applied field is shown in Fig. 3, where the result for the sample: glass/100 $\AA \mathrm{Co} /(\mathrm{Co} 25 / \mathrm{Cu} 18) 10$ is presented. We have probed the magnetoresistance as a function of $\mathrm{Cu}$ spacer thickness around the second $\mathrm{AF}$ peak for glass $/ 100 \AA \mathrm{Co} /(\mathrm{Co} 25 / \mathrm{CuX}) 10$ series and the highest value was found for the sample with $X=21 \AA$ spacer thickness: $6.5 \%$ at room temperature and $16 \%$ at $8 \mathrm{~K}$. Taking into account the shunting effect of the buffer layer increases these values to $7.8 \%$ and $19.2 \%$, respectively.

\section{Conclusions}

Structural and magnetic correlations were studied in polycrystalline $\mathrm{Co} / \mathrm{Cu}$ multilayers deposited by the MBE technique on glass substrates. It was shown that the GMR effect is present in the samples with non-epitaxial growth but its magnitude is closely related to the roughness of the interfaces. The influence of different buffer layers with different thicknesses was investigated. The best results were obtained for multilayers grown on $100 \AA$ cobalt buffer layer for which the highest value of GMR was measured. The same sample exhibited the lowest interface roughness. The same oscillatory character of AF coupling as for sputtered films was found with oscillations period of about $10 \AA$ as predicted by the theory, so 
a lack of oscillations and/or GMR effect observed by some authors in MBE-grown $\mathrm{Co} / \mathrm{Cu}$ thin films cannot be attributed to the deposition technique. However, the GMR effect values are higher for the sputtered than for the MBE-grown samples and the origin of this difference is still not clear.

\section{Acknowledgments}

This work was partially supported by the grant No. 8T11B 05309 and grant No. 2P03B 02109 from the Committee for Scientific Research. The magnetoresistance measurements were performed using the setup constructed within the framework of the grant No. 2238892 03. The Philips MRD diffractometer used in this work was founded by the Foundation for Polish Science within the program SEZAM'94.

\section{References}

[1] A. Schreyer, K. Brohl, J. Anker, C. Majkrzak, T. Zeidler, P. Brodeker, N. Metoki, H. Zabel, Phys. Rev. B 47, 15334 (1993).

[2] J.P. Renard, P. Beauvillain, C. Dupas, K.Le Dong, P. Veillet, E. Velu, C. Marliere, D. Renard, J. Magn. Magn. Mater. 115, L147 (1992).

[3] W. Egelhoff, M. Kieff, Phys. Rev. B 45, 7795 (1992).

[4] U. Gradman, H. Elmer, J. Kohlhepp, Mat. Res. Soc. Symp. Proc. 313, 107 (1993).

[5] S. Parkin, R. Bhadra, K. Roche, Phys. Rev. Lett. 66, 2152 (1991).

[6] L.G. Parratt, Phys. Rev. 95, 359 (1954).

[7] M. Aleszkiewicz, R. Kalinowski, R. Czajka, W. Polewska, A. Wawro, L.T. Baczewski, J. Raułuszkiewicz, Acta Phys. Pol. A 91, (1997).

[8] M. Howson, B. Hickey, J. Xu, D. Greig, J. Magn. Magn. Mater. 126, 416 (1993). 